\title{
Synthesis and Characterization of Photocurable Elastomers from Poly(glycerol-co-sebacate)
}

\author{
Christiaan L. E. Nijst, ${ }^{\dagger, \ddagger}$ Joost P. Bruggeman, ${ }^{\dagger, \S}$ Jeffrey M. Karp,,$"$ Lino Ferreira, ${ }^{\dagger, \perp}$ \\ Andreas Zumbuehl, ${ }^{\dagger}$ Christopher J. Bettinger, ${ }^{\dagger}$ and Robert Langer ${ }^{*},+, \|$ \\ Department of Chemical Engineering and Harvard-MIT Division of Health Science and Technology, \\ Massachusetts Institute of Technology, Cambridge, Massachusetts 02139, Department of Biomedical \\ Engineering, Eindhoven University of Technology, 5600 MB Eindhoven, The Netherlands, Department of \\ Plastic and Reconstructive Surgery, Erasmus Medical Center, Erasmus University Rotterdam, 3015 GE \\ Rotterdam, The Netherlands, and Center of Neurosciences and Cell Biology, University of Coimbra, \\ 3004-517 Coimbra, and Biotechnology Innovation Center, 3060-197 Cantanhede, Portugal
}

Received April 18, 2007; Revised Manuscript Received June 29, 2007

\begin{abstract}
Elastomeric networks are increasingly being investigated for a variety of biomedical applications including drug delivery and tissue engineering. However, in some cases, their preparation requires the use of harsh processing conditions (e.g., high temperature), which limits their biomedical application. Herein, we demonstrate the ability to form elastomeric networks from poly(glycerol-co-sebacate) acrylate (PGSA) under mild conditions while preserving a wide range of physical properties. These networks presented a Young's modulus between 0.05 and $1.38 \mathrm{MPa}$, an ultimate strength from 0.05 to $0.50 \mathrm{Mpa}$, and elongation at break between $42 \%$ and $189 \%$ strain, by varying the degree of acrylation (DA) of PGSA. The in vitro enzymatic and hydrolytic degradation of the polymer networks was dependent on the DA. The copolymerization of poly(ethylene glycol) diacrylate with PGSA allowed for an additional control of mechanical properties and swelling ratios in an aqueous environment, as well as enzymatic and hydrolytic degradation. Photocured PGSA networks demonstrated in vitro biocompatibility as judged by sufficient human primary cell adherence and subsequent proliferation into a confluent monolayer. These photocurable degradable elastomers could have potential application for the encapsulation of temperaturesensitive factors and cells for tissue engineering.
\end{abstract}

\section{Introduction}

The development of biodegradable elastomers has increasingly become important in biomedical applications. Elastomers have gained popularity because they can provide stability and structural integrity within a mechanically dynamic environment without irritation to the hosting tissues ${ }^{1,2}$ while they exhibit mechanical properties similar to those of soft tissues..$^{2-5}$ Biodegradable elastomers can be important materials for a wide variety of medical applications including drug delivery and tissue regeneration, where (cell-seeded) constructs are designed to aid or replace damaged or diseased tissue. ${ }^{2-4}$ Examples of applications for elastomeric biodegradable biomaterials are smalldiameter vascular grafts ${ }^{6,7}$ and nerve conduits. ${ }^{8-10}$ Current biodegradable elastomers include poly(glycerol-co-sebacate) ${ }^{2}$ poly(citric-co-diol) ${ }^{4}$ star poly ( $\epsilon$-caprolactone- $c o$-D,L-lactide), ${ }^{5,11}$ poly(trimethylene carbonate-co- $\epsilon$-caprolactone), ${ }^{12}$ and poly(trimethylene carbonate- $c o$-D,L-lactide). ${ }^{13}$

We recently created a tough biodegradable elastomer, poly(glycerol sebacate) (PGS), which features robust mechanical properties and in vitro and in vivo biocompatibility. ${ }^{2,14}$ However, harsh conditions $\left(>80{ }^{\circ} \mathrm{C},<5 \mathrm{~Pa}\right)$ and long reaction times

* To whom correspondence should be addressed. E-mail: rlanger@mit.edu. Phone: (617) 253-3107. Fax: (617) 253-8827.

$\dagger$ Department of Chemical Engineering, Massachusetts Institute of Technology.

$\doteqdot$ Eindhoven University of Technology.

$\S$ Erasmus University Rotterdam.

"Harvard-MIT Division of Health Science and Technology, Massachusetts Institute of Technology.

${ }^{\perp}$ University of Coimbra and Biotechnology Innovation Center. (typically $>24 \mathrm{~h}$ ) are required for its curing and thus limit its ability to polymerize directly in a tissue or to incorporate cells or temperature-sensitive molecules. As such, there is an unmet need to develop alternative processing strategies to overcome the limitations of thermally processing PGS. One convenient strategy is the implementation of photopolymerization. This technique has been utilized for several decades in biomedical research and has become an integral method for in situ delivery of resins in the practice of dentistry. ${ }^{15-17}$ Recently, there has been great interest in using photopolymerization techniques to prepare polymeric networks for tissue engineering applications as well as for minimally invasive medical procedures. ${ }^{18,19} \mathrm{To}$ this end, acrylate groups have been included in polymers for participation in chemical cross-linking between polymer chains by photoinduced free radical polymerization. ${ }^{11,20-22}$

In this paper, we describe the synthesis and characterization of a photocurable polymer based on the chemical modification of PGS with acrylate moieties (designated poly(glycerol sebacate) acrylate, or PGSA). PGSA can be cured rapidly (within minutes) at ambient temperatures to form polymeric networks with a wide range of mechanical properties and in vitro enzymatic degradation and hydrolysis profiles. Incorporation of poly(ethylene glycol) diacrylate (PEG-DA) allowed for additional control of mechanical properties and swelling ratios in an aqueous environment. Initial experiments with photocured PGSA networks demonstrated in vitro biocompatibility by sufficient cell adherence and subsequent proliferation into a confluent cell monolayer. 


\section{Experimental Section}

2.1. Synthesis of PGSA. All chemicals were purchased from SigmaAldrich (Milwaukee, WI), unless stated otherwise. PGS was synthesized according to previously published methods. ${ }^{2}$ Briefly, the PGS prepolymer was synthesized by polycondensation of equimolar glycerol and sebacic acid (Fluka, Buchs, Switzerland) at $120^{\circ} \mathrm{C}$ under argon for 24 $\mathrm{h}$ before reduction of the pressure from 1 Torr to $40 \mathrm{mTorr}$ over $5 \mathrm{~h}$. The polycondensation was continued for another $24 \mathrm{~h}$. This material was used without any further purification. The PGS prepolymer was acrylated in the following manner: a flame-dried round-bottom flask was charged with PGS prepolymer ( $20 \mathrm{~g}$, with $78 \mathrm{mmol}$ of hydroxyl groups), $200 \mathrm{~mL}$ of anhydrous dichloromethane, and 4-(dimethylamino)pyridine (DMAP) $(20 \mathrm{mg}, 0.18 \mathrm{mmol})$. The reaction flask was cooled to $0{ }^{\circ} \mathrm{C}$ under a positive pressure of $\mathrm{N}_{2}$. Acryloyl chloride $(0.25-0.80$ $\mathrm{mol} / \mathrm{mol}$ of hydroxyl groups on the PGS prepolymer) was slowly added parallel to an equimolar amount of triethylamine. The reaction was allowed to reach room temperature and was stirred for an additional $24 \mathrm{~h}$. The resulting mixture was dissolved in ethyl acetate, filtered, and dried at $45^{\circ} \mathrm{C}$ and $5 \mathrm{~Pa}$.

2.2. Polymer Characterization. ${ }^{1} \mathrm{H}$ nuclear magnetic resonance $\left({ }^{1} \mathrm{H}\right.$ NMR) spectra of the PGS prepolymer and PGSA were recorded (Varian Unity-300 NMR spectrometer). Chemical shifts were referenced relative to the peak for $\mathrm{CDCl}_{3}$ at $7.27 \mathrm{ppm}$. The chemical composition was determined by calculating the signal integrals of $-\mathrm{COCH}_{2} \mathrm{CH}_{2} \mathrm{CH}_{2}-$ at $1.2,1.5$, and $2.2 \mathrm{ppm}$ for sebacic acid, $-\mathrm{CH}_{2} \mathrm{CH}-$ at 3.7, 4.2, and $5.2 \mathrm{ppm}$ for glycerol, and $-\mathrm{CH}=\mathrm{CH}_{2}$ at $5.9,6.1$, and $6.5 \mathrm{ppm}$ for the protons on the acrylate groups. The signal intensity of the methylene groups of sebacic acid (1.2 ppm) and the acrylate groups (average signal intensities of 5.9, 6.1, and $6.5 \mathrm{ppm}$ ) were used to calculate the DA. The molecular weights of the PGS prepolymer and PGSA were determined by gel permeation chromatography (GPC) using THF on Styragel columns (series of HR-4, HR-3, HR-2, and HR-1, Waters, Milford, MA).

2.3. Preparation and Characterization of Photocured PGSA. PGSA networks were formed by mixing PGSA with $0.1 \%(\mathrm{w} / \mathrm{w})$ photoinitiator (2,2-dimethoxy-2-phenylacetophenone), and the polymerization reaction was initiated by exposure to ultraviolet light (ca. 4 $\mathrm{mW} / \mathrm{cm}^{2}$, model 100AP, Blak-Ray) for $10 \mathrm{~min}$. Attenuated total reflectance Fourier transform infrared (ATR-FTIR) spectroscopy analysis was performed on a Nicolet Magna-IR 500 spectrophotometer to confirm the cross-link reaction. For that purpose, thermally cured PGS and photocured PGSA slabs, PGS prepolymer, and PGSA dissolved in chloroform were placed on top of a $\mathrm{ZnSe}$ crystal. The thermal properties of disks from thermally cured PGS, photocured PGSA (DA of 0.31 and 0.54 ), and photocured PGSA $(\mathrm{DA}=0.34$ ) containing 5\% PEG diacrylate were characterized using differential scanning calorimetry (DSC; DSC Q 1000), for two cycles, within the temperature range from -90 to $+250{ }^{\circ} \mathrm{C}$ using a heating/cooling rate of $10{ }^{\circ} \mathrm{C} / \mathrm{min}$. Tensile tests were conducted using an Instron 5542 (according to ASTM standard D412-98a) on dog-bone-shaped polymer strips $(115 \times 25 \times 1.2 \mathrm{~mm})$ cut from photocured PGSA sheets. The photocured networks were first soaked in $100 \%$ ethanol for $24 \mathrm{~h}$ and subsequently soaked in PBS for $24 \mathrm{~h}$ prior to mechanical testing. The strain rate was $50 \mathrm{~mm} / \mathrm{min}$, and all samples were elongated to failure. The sol content was measured by the mass differential after incubation of the PGSA samples in $100 \%$ ethanol for $24 \mathrm{~h}$. Swelling by hydration was measured by the mass differential after incubation of the PGSA samples in PBS for $24 \mathrm{~h}$. The mass density was measured with a pycnometer (Humboldt Manufacturing Co.). The density and Young modulus of the samples were used to calculate the cross-linking density and relative molecular mass between cross-links $\left(M_{\mathrm{c}}\right)$ as described previously. ${ }^{2}$

2.4. Copolymerization of PEG Diacrylate and PGSA. Networks of PGSA-PEG diacrylate were prepared by mixing $10 \%, 50 \%$, and $90 \%(\mathrm{w} / \mathrm{w})$ PGSA $(\mathrm{DA}=0.34)$ with PEG diacrylate $\left(M_{\mathrm{w}}=700\right)$ including $0.1 \%(\mathrm{w} / \mathrm{w})$ photoinitiator, followed by photopolymerization under UV light for $10 \mathrm{~min}$. Poly(ethylene glycol) polymers were prepared from PEG diacrylate (liquid) containing $0.1 \%(\mathrm{w} / \mathrm{w})$ photoinitiator, followed by photopolymerization. The swelling ratio in PBS was determined by the mass differential.

2.5. In Vitro Degradation. Degradation rates via hydrolysis were measured by incubating sol-free samples $(n=4)$ of PGS, photocured PGSA (DA of 0.31 and 0.54 ), and photocured PGSA containing 5\% PEG diacrylate in $20 \mathrm{~mL}$ of $0.1 \mathrm{mM} \mathrm{NaOH}$ at $37{ }^{\circ} \mathrm{C}$. The samples were removed, washed in $\mathrm{ddH}_{2} \mathrm{O}$, dried at $90{ }^{\circ} \mathrm{C}$ for 7 days, and weighed again to determine the mass loss. In the enzyme degradation study, these samples $(n=4)$ were degraded in vitro in PBS in the presence of bovine pancreatic cholesterol esterase ( 40 units $/ \mathrm{mL}$ ) and incubated at $37{ }^{\circ} \mathrm{C}$. The samples were removed, washed in $\mathrm{ddH}_{2} \mathrm{O}$, dried at 90 ${ }^{\circ} \mathrm{C}$ for 7 days, and weighed again to determine the mass loss.

2.6. In Vitro Cell Attachment and Proliferation. Primary human foreskin fibroblasts (HFFs; ATCC, Manassas, VA) were cultured in high-glucose Dulbecco's minimal essential medium (DMEM) supplemented with $10 \%(\mathrm{v} / \mathrm{v})$ fetal bovine serum (Invitrogen), $100 \mu \mathrm{g} / \mathrm{mL}$ streptomycin (Invitrogen), and $100 \mathrm{U} / \mathrm{mL}$ penicillin (Invitrogen) (further denoted as growth medium) at $37{ }^{\circ} \mathrm{C}$ and $5 \% \mathrm{CO}_{2}$. Cells between passages four and six were harvested by using trypsin $(0.025 \%)$ /EDTA $(0.01 \%)$ and quenched with an equal volume of growth medium to resuspend the cells. Photocured PGSA $(\mathrm{DA}=0.34)$ covered glass slides (diameter $18 \times 1 \mathrm{~mm}, n=3$ ) were prepared by spin-coating $20 \%$ $(\mathrm{w} / \mathrm{v})$ PGSA in dimethyl sulfoxide (DMSO) at $3400 \mathrm{rpm}$ for $5 \mathrm{~min}$ followed by a $10 \mathrm{~min}$ UV polymerization. The photocured spin-coated PGSA disks were incubated with growth medium in a 12-well plate for $4 \mathrm{~h}$ to remove photoinitiator, residual DMSO, and any unreacted monomers prior to seeding. Each disk was seeded with $5000 \mathrm{cells} / \mathrm{cm}^{2}$ using $2 \mathrm{~mL}$ of growth medium. The cell attachment was evaluated after the cell-seeded constructs were lightly washed with PBS after $4 \mathrm{~h}$, and the cell density was assessed and compared to that of cell attachment on tissue-cultured polystyrene (TCPS). Subsequently, at predetermined times, these cell cultures were washed with PBS and fixed with $4 \%$ formaldehyde solution for $10 \mathrm{~min}$, and the cell density was determined by counting the cells from randomly picked equally sized areas $(0.005$ $\mathrm{cm}^{2}, n=9$ ). Phase micrographs of the cells were taken at $10 \times$ magnification, and the cell perimeter and cell area were calculated using Axiovision software (Zeiss, Germany). For the cell morphology measurements, each data set consists of measurements of at least 100 cells across several images from randomly picked areas. Scanning electron microscopy (SEM) images were taken after culture substrates were washed three times in PBS and fixed with $4 \%(\mathrm{v} / \mathrm{v})$ paraformaldehyde in PBS. After being rinsed with PBS buffer three times, the dishes were dehydrated in graded alcohols $(50 \%, 70 \%, 80 \%, 90 \%, 95 \%$, and $100 \%$ ) and then air-dried in HMDS. The samples were then sputtercoated with platinum and examined on a JEOL JSM-5910 scanning electron microscope.

\section{Results and Discussion}

3.1. Polymer Characterization. PGSA was prepared by the acrylation of PGS prepolymer (Figure 1A,B). The PGS prepolymer had a weight-average molecular weight $\left(M_{\mathrm{w}}\right)$ of 23000 , a number-average molecular weight $\left(M_{\mathrm{n}}\right)$ of 6500 as determined by GPC, and a polydispersity index (PDI) of 3.5. The molar composition of the PGS prepolymer was approximately 1:1 glycerol/sebacic acid as confirmed by ${ }^{1} \mathrm{H}$ NMR analyses (Figure 2A). The incorporation of acrylate groups was confirmed by ${ }^{1} \mathrm{H}$ NMR by the appearance of the peaks at $\delta 5.9,6.1$, and 6.4 ppm (Figure 2B). This was also confirmed by ATR-FTIR by the appearance of an absorption band at $1375 \mathrm{~cm}^{-1}$, corresponding to the $-\mathrm{CH}$ stretching of the secondary alkyl group related to the acrylate groups ${ }^{23}$ and known not to be present in poly(acryloyl chloride) ${ }^{24}$ (Figure 2C). Typically, $66 \%$ of the acryloyl chloride added in the experiment was incorporated in the prepolymer as calculated from signal intensities of ${ }^{1} \mathrm{H}$ NMR 
A<smiles>CCC(C)(C)CC(O)COC(=O)CC(C)(C)C(=O)OCC(C)(C)C(=O)O</smiles>

B

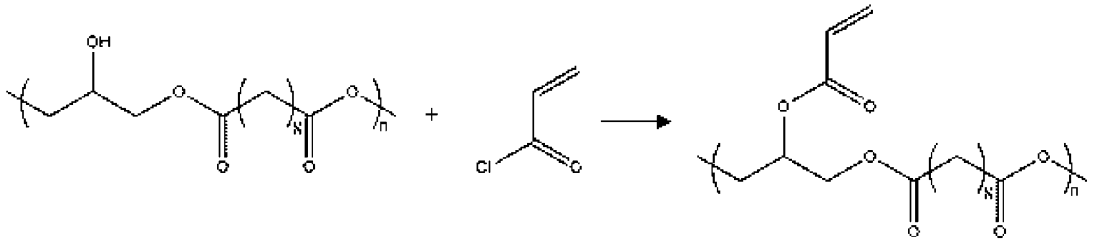

C

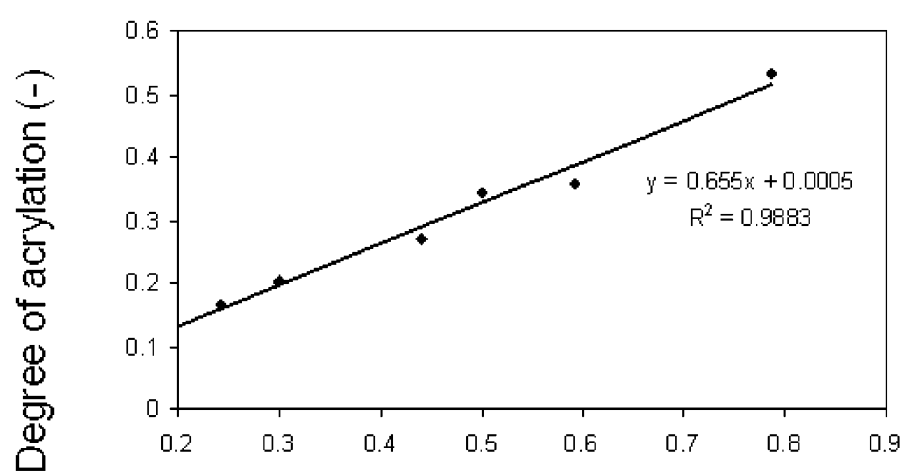

Mol acryloyl chloride per mol of glycerol-sebacate (-)

Figure 1. (A) Polycondensation of glycerol and sebacic acid, yielding the PGS prepolymer. R represents $\mathrm{H}$ or the polymer chain. (B) Acrylation of the prepolymer (not all binding possibilities are shown). To simplify the scheme, $100 \%$ conversion is shown. The hydroxyl groups in the PGS are the preferred site of acrylation. The acrylation of carboxylic acid groups occurs to a much lesser degree. (C) Increasing the molar equivalent of acryloyl chloride increases the degree of acrylation linearly.

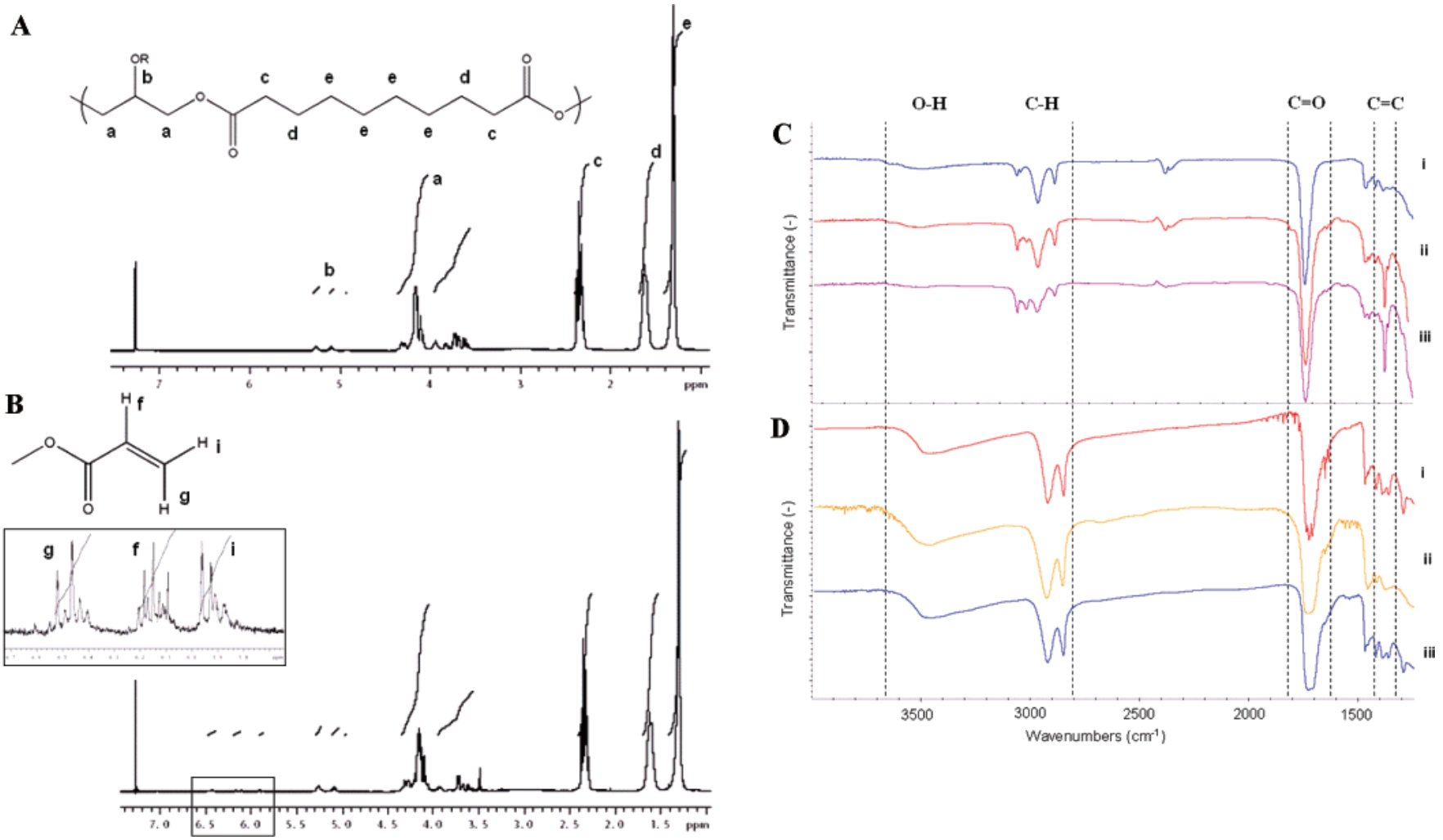

Figure 2. ${ }^{1} \mathrm{H}$ NMR spectrum of (A) PGS prepolymer and (B) PGSA. The sebacic acid and glycerol in the polymer matrix were identified at 1.2 , 1.5, and $2.2 \mathrm{ppm}$ and 3.7, 4.2, and $5.2 \mathrm{ppm}$ by hydrogens located on the carbons labeled "a"-"e". Vinyl groups located on the PGSA were identified at 5.9, 6.1, and 6.4 ppm labeled "f"-“i". (C) ATR-FTIR spectra of PGS prepolymer (C,i), PGSA (DA of 0.20) (C,ii), PGSA (DA =0.54) $(C$, iii), thermally cured PGS $(D, i)$, photocured PGSA $(D A=0.20)(D$,ii), and photocured PGSA $(D A=0.54)(D$,iii). The absorption band at 1375 $\mathrm{cm}^{-1}$ associated with acrylate groups (spectra in $(C$, ,ii) and $(C$, iii)) disappeared after the photopolymerization reaction (spectra in $(D, i i)$ and $(\mathrm{D}, \mathrm{iii}))$. 
(Figure 1C). PGSA with a DA between 0.17 and 0.54 was obtained. The ${ }^{1} \mathrm{H}$ NMR data show that acryloyl chloride reacts preferentially with the hydroxyl groups of glycerol compared to the carboxylate groups of sebacic acid. This was confirmed by the increase of the signal integral at $\delta 5.2 \mathrm{ppm}$, corresponding to the resonance of protons from the trisubstituted glycerol, and the decrease of the signal integral at ca. $\delta 3.7 \mathrm{ppm}$, corresponding to the resonance of protons from monosubstituted glycerol (Figure 2A,B), with the increment of the DA. ${ }^{1} \mathrm{H}-{ }^{1} \mathrm{H}$ COSY NMR and quantitative ${ }^{13} \mathrm{C}$ NMR analysis showed minimal $(<5 \%)$ substitution of terminal carboxylate groups (data not shown). As determined by GPC analysis, the $M_{\mathrm{w}}$ of the PGSA remained unchanged after acrylation (data not shown). The PGSA prepolymer is not soluble in aqueous solutions but is soluble in most organic solvents such as ethanol, dimethyl sulfoxide, benzene, tetrahydrofuran, acetyl acetate, dichloromethane, and dimethylformamide.

3.2. Characterization of Photocured PGSA. The polymerization of PGSA using UV light in the presence of the photoinitiator 2-dimethoxy-2-phenylacetophenone yields elastomeric networks. ATR-FTIR analysis of all photocured PGSA elastomers (Figure 2C,D) showed typical absorption bands of hydroxyl $\left(3500-3200 \mathrm{~cm}^{-1}\right)$ and ester $\left(1800-1600 \mathrm{~cm}^{-1}\right)$ groups in the polymer backbone, as was observed for thermally cured PGS. The broad peak at $3475 \mathrm{~cm}^{-1}$ is assigned to hydrogen-bonded hydroxyl groups, likely from free hydroxyl groups which are not modified by acryloyl chloride. The formation of a polymer network after photocuring of PGSA was confirmed by the increase of the band at $2930 \mathrm{~cm}^{-1}$, corresponding to the vibration of alkyl groups, and the elimination of the band at $1375 \mathrm{~cm}^{-1}$, known to be associated with acrylate groups. $^{23}$

The $T_{\mathrm{g}}$ values of thermally cured PGS, photocured PGSA $(\mathrm{DA}=0.31,0.54)$, and photocured PGSA $(\mathrm{DA}=0.34)$ containing 5\% PEG diacrylate were respectively $-28.1,-32.2$, -31.1 , and $-31.4{ }^{\circ} \mathrm{C}$. These results indicate that all polymers and copolymers are in a rubbery state at $37^{\circ} \mathrm{C}$.

Currently, the mechanical properties of thermally cured PGS and other similar polymers can be controlled by altering the processing conditions or by changing the molecular weight of the difunctional precursors., ${ }^{2,4}$ The introduction of acrylate groups into the prepolymer facilitated an additional level of control. Specifically, Young's modulus and the ultimate tensile strength of the photocured PGSA were linearly proportional to the DA (Figure 3A,B), and no permanent deformations were observed after mechanical testing. The mechanical properties of the photocured PGSA spanned from soft to relatively stiff as determined by the tensile Young modulus of the polymer, which varied from $0.05 \mathrm{MPa}(\mathrm{DA}=0.17)$ to $1.38 \mathrm{MPa}(\mathrm{DA}=0.54)$. This demonstrates the potential to achieve the mechanical compliance of, for instance, the peripheral nerve, which has a Young modulus of approximately $0.45 \mathrm{MPa}{ }^{25}$ The ultimate tensile strength ranged from 0.05 to $0.50 \mathrm{MPa}$ (Figure 3B), whereas the strain to failure of photocured PGSA ranged from $170 \%$ to $47.4 \%$ with increasing DA (Figure $3 \mathrm{~A}$ ).

The degree of swelling of the elastomeric networks in ethanol and water ranged from $50 \%$ to $70 \%$ and from $8 \%$ to $12 \%$, respectively, and did not change appreciably as a function of the DA (Figure 3C). The high degree of swelling in ethanol facilitates removal of unreacted monomers (sol content) or potential incorporation of specific factors. The low degree of swelling in water may help to maintain the mechanical properties once implanted. The sol content of the polymer decreased from $40 \%$ to $<10 \%$ by increasing the DA from 0.20 to 0.54 (Figure
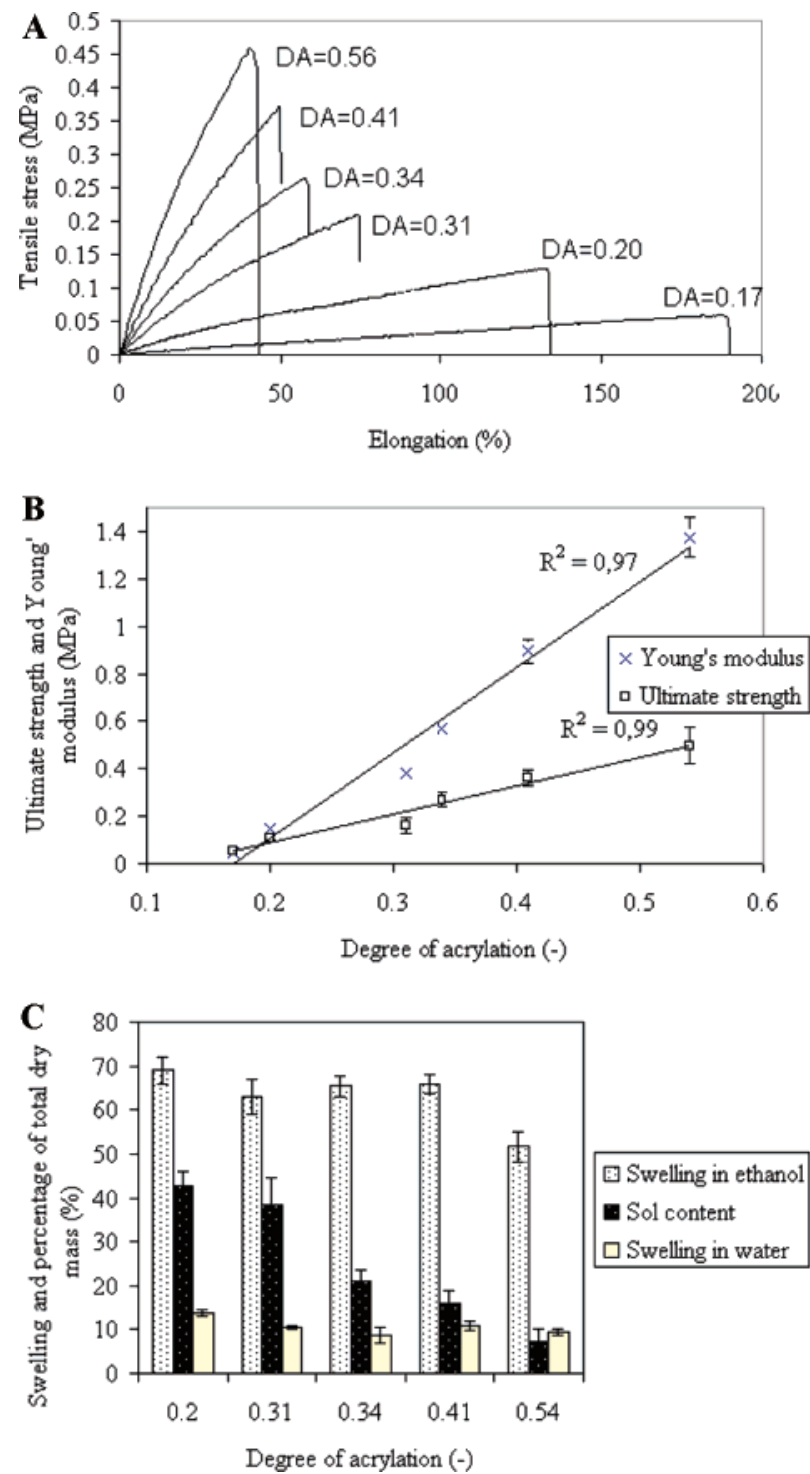

Figure 3. (A) Stress-strain curves of photocured PGSA elastomer from a representative experiment $(n=4)$. (B) Through increasing the degree of acrylation, the ultimate tensile strength and Young modulus increased linearly. (C) Degree of swelling in ethanol and water and sol content as a function of the degree of acrylation.

$3 C)$. This is likely due to the increasing number of new crosslinks between the polymer chains. The high sol content, which was achieved with a lower DA, may be unfavorable for in situ polymerization where unreacted acrylated macromers would diffuse into the surrounding tissue. The mechanical properties, however, are linearly proportional to the DA and are correlated to the formation of new cross-links within the polymer network and not to the unreacted macromers in the network.

The density of the photocured elastomers decreased slightly with increasing DA (Table 1), which is similar to other thermally cured elastomers in which the density is inversely proportional to the curing time. ${ }^{26}$ The cross-linking density and relative molecular mass between cross-links $\left(M_{\mathrm{c}}\right)$ were calculated using the density and Young modulus of the samples (Table 1) as previously described. ${ }^{2}$ By increasing the DA in photocured PGSA from 0.17 to 0.54 , the cross-linking density increased from 6.4 to $185 \mathrm{~mol} / \mathrm{m}^{3}$ and the relative molecular mass between cross-links decreased from 18000 to 600 .

3.3. Copolymerization of PGSA with PEG Diacrylate. Another advantage of photocurable PGSA is that it can be easily combined with other acrylated precursors to achieve a wider 
Table 1. Physical and Mechanical Properties of PGSA Networks ${ }^{a}$

\begin{tabular}{ccccccc}
\hline $\begin{array}{c}\text { degree of } \\
\text { acrylation }\end{array}$ & density $\left(\mathrm{g} / \mathrm{cm}^{3}\right)$ & $\begin{array}{c}\text { Young's } \\
\text { modulus }(\mathrm{MPa})\end{array}$ & $\begin{array}{c}\text { enlongation } \\
(\%)\end{array}$ & $\begin{array}{c}\text { ultimate } \\
\text { strength }(\mathrm{MPa})\end{array}$ & $\begin{array}{c}\text { rel molecular mass } \\
\text { cross-linking } \\
\text { density }\left(\mathrm{mol} / \mathrm{m}^{3}\right)\end{array}$ & $\begin{array}{c}\text { between cross-links } \\
\left(M_{\mathrm{c}}\right)(\mathrm{g} / \mathrm{mol})\end{array}$ \\
\hline 0.17 & $1.21\{0.02\}$ & $0.048\{0.005\}$ & $170\{17.2\}$ & $0.054\{0.005\}$ & $6.4\{0.7\}$ & $18906\{232\}$ \\
0.20 & $1.19\{0.02\}$ & $0.148\{0.004\}$ & $101\{26.5\}$ & $0.109\{0.011\}$ & $19.8\{0.6\}$ & $6013\{253\}$ \\
0.31 & $1.16\{0.02\}$ & $0.383\{0.028\}$ & $54.7\{14.1\}$ & $0.163\{0.034\}$ & $51.5\{3.9\}$ & $2262\{185\}$ \\
0.34 & $1.15\{0.01\}$ & $0.568\{0.222\}$ & $60.01\{5.73\}$ & $0.270\{0.032\}$ & $76.4\{3.0\}$ & $1514\{73.3\}$ \\
0.41 & $1.15\{0.02\}$ & $0.895\{0.052\}$ & $51.1\{7.41\}$ & $0.364\{0.034\}$ & $120.4\{7.0\}$ & $953.9\{69.1\}$ \\
0.54 & $1.15\{0.01\}$ & $1.375\{0.084\}$ & $47.4\{11.3\}$ & $0.498\{0.079\}$ & $185\{11.3\}$ & $620.1\{42.4\}$ \\
\hline
\end{tabular}

a Values are reported as the mean followed by the standard deviation in braces.

range of material properties. As an example, we chose to copolymerize PGSA with PEG diacrylate $(0.7 \mathrm{kDa})$, a hydrophilic nondegradable polymer. The effect on the physical properties (Young's modulus, ultimate strength, elongation, and swelling ratio in water) was studied of elastomers formed by changing the weight percentage of PEG diacrylate with PGSA $(\mathrm{DA}=0.34)$ (Figure 4). Specifically, by increasing the concentration of PEG diacrylate, the elongation ranged from $60 \%$ to $4 \%$, Young's modulus from 0.6 to $20 \mathrm{MPa}$, and the ultimate strength from 0.270 to $0.890 \mathrm{MPa}$. The elastomer formed by the copolymerization of PEG diacrylate with PGSA $(\mathrm{DA}=0.34)$ mixed with equal masses, 50:50 PGSA-PEG, showed a 10-fold higher Young's modulus and ultimate strength than the 95:05 PGSA-PEG elastomer, while its elongation was maintained. Furthermore, the swelling behavior of these networks could be tuned from $45 \%$ to $10 \%$ by changing the concentration of PEG diacrylate from $90 \%$ to $0 \%$.

3.4. In Vitro Degradation of Photocured PGSA. Previous studies have shown that the degradation of PGS in vitro is difficult to correlate with the in vivo degradation. ${ }^{2,14}$ PGS degrades $15 \%$ in 10 weeks in PBS, whereas full degradation is observed after 6 weeks in vivo. Similar to PGS, PGSA (DA = 0.31 ) degraded only $10 \%$ after 10 weeks in PBS (data not shown). Given this discrepancy between in vitro degradation and in vivo degradation results, we performed in vitro hydrolysis and enzymatic degradation studies to examine the relative differences in mass loss over time between the different polymer networks. The polymer networks that were examined in these degradation studies were thermally cured PGS, photocured PGSA with 2 degrees of acrylation (low degree of acrylation, PGSA-LA (DA $=0.31)$, and high degree of acrylation, PGSAHA $(\mathrm{DA}=0.54)$ ), and PGSA-PEG (PGSA-LA copolymerized with $5 \%$ PEG diacrylate).

In the enzyme degradation study, these polymers were degraded in vitro in PBS in the presence of bovine pancreatic cholesterol esterase (40 units/mL). Pancreatic cholesterol esterase has been shown to be identical to the esterases associated with macrophages that are known to degrade polyesters. ${ }^{27-29}$ PGS and PGSA-LA showed mass loss over time, whereas PGSA-HA and PGSA-PEG did not. PGS degraded by $60 \%$ over 48 h, while PGSA-LA, which has a lower cross-linking density, only degraded by $40 \%$ (Figure 5A). These results suggest that the alkyl cross-links formed in PGSA networks by the acrylate groups may be less susceptible to cholesterol esterase than the polymer networks with only ester cross-links, as formed in PGS networks.

The hydrolysis study was performed in a sodium hydroxide $(0.1 \mathrm{mM})$ solution as described previously. ${ }^{4,26}$ The mass loss profile via hydrolysis of photocured PGSA-LA and PGSA-HA was similar to that of PGS (Figure 5B). However, the absolute mass loss of PGSA-LA was significantly $(P<0.01)$ higher compared to those of PGS and PGSA-HA in all time points. Interestingly, the rate of mass loss of PGSA-PEG was significantly $(P<0.01)$ lower compared to those of PGS and PGSA-HA, and the mass loss revealed a more linear profile. Hence, copolymerization of PGSA-LA with 5\% PEG diacrylate

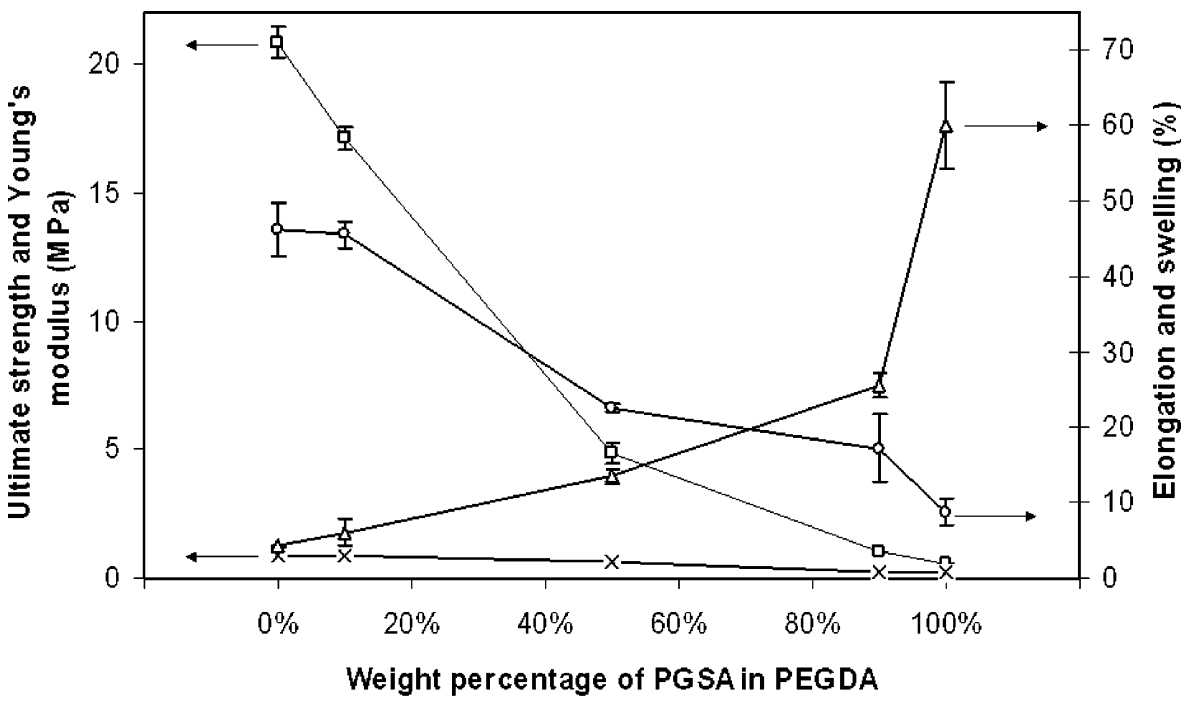

$-\square-$ Young's modulus $-x-$ Ultimate strength $\neg-$ Elongation $\multimap-S$ welling in water

Figure 4. Copolymerization of PGSA (DA $=0.34)$ and PEG diacrylate $\left(M_{w}=700\right)$ where PEG chains become incorporated as cross-links within the PGSA network. Increasing the concentration of PGSA decreases the Young modulus, ultimate strength, and swelling in water, whereas the elongation increases. 

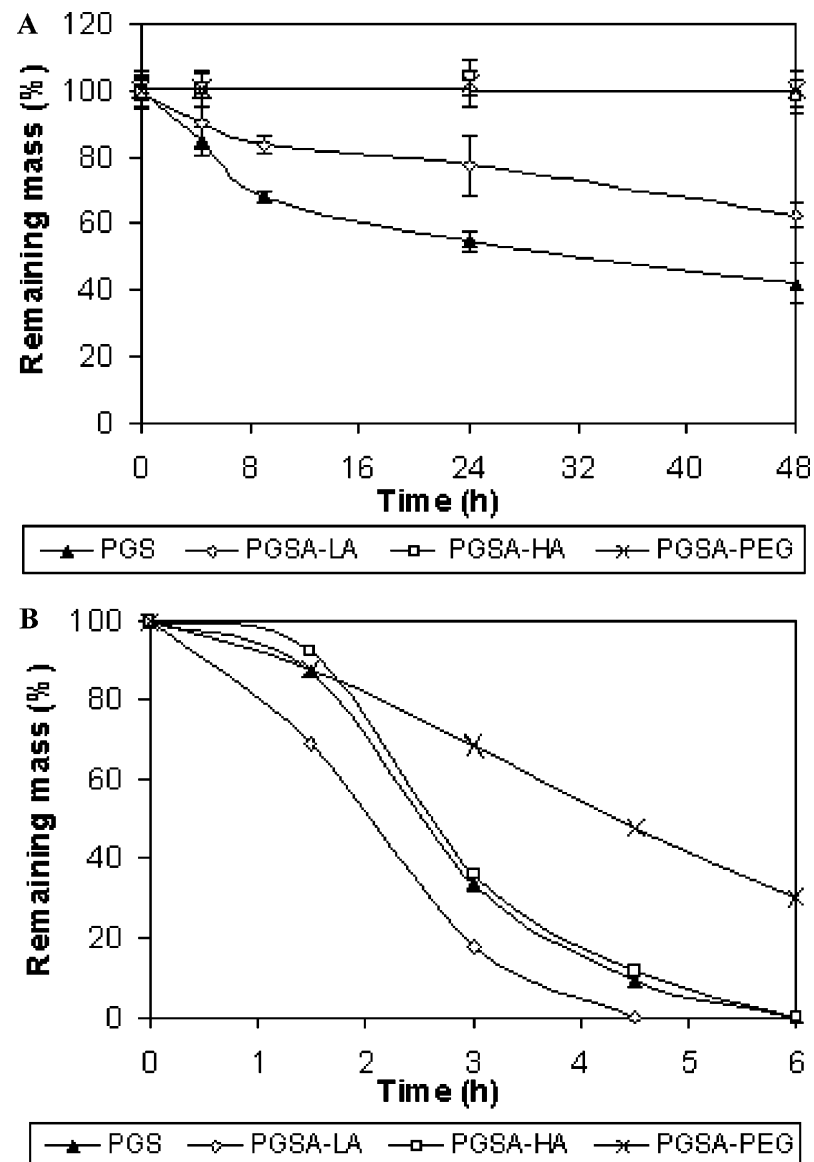

Figure 5. (A) In vitro enzymatic degradation of PGS, photocured PGSA (DA $=0.31,0.54$ ), and PGSA (DA $=0.34+5 \%$ PEG diacrylate) in bovine cholesterol esterase $\left(\mathrm{pH} 7.2,37^{\circ} \mathrm{C}\right)(n=3)$ for $4,8,24$, and $48 \mathrm{~h}$. (B) In vitro hydrolytic degradation of PGS, photocured PGSA (DA $=0.31,0.54$ ), and PGSA (DA $=0.34,+5 \%$ PEG diacrylate) in $\mathrm{NaOH}(0.1 \mathrm{mM})(n=3)$ for $0,1.5,3,4.5$, and $6 \mathrm{~h}$ at $37^{\circ} \mathrm{C}$. resulted in elastomers with mechanical properties (Figure 4) similar to those of PGSA-HA, but showed a much slower degradation rate via hydrolysis when compared to photocured PGSA and PGS (Figure 5B). These results show that the in vitro hydrolytic degradation rate of photocured PGSA can be decreased, independent of the initial mechanical strength.

3.5. In Vitro Cell Adhesion and Proliferation on Photocured PGSA. Our initial in vitro biocompatibility study showed that the photocured elastomers support cell adhesion and proliferation: $59 \pm 12 \%$ of the primary human foreskin fibroblasts seeded on photocured PGSA attached after $4 \mathrm{~h}$, as compared to the attachment on TCPS, which we set at $100 \%$. The cells were viable, assessed by trypan blue staining, as only $1.4 \%( \pm 0.5 \%)$ of the cells were positive. The attached cells showed a relatively normal morphology, as displayed in Figure $6 \mathrm{~A}-\mathrm{C}$. Figure $6 \mathrm{~B}$ shows a detailed SEM image of cell spreading $(\mathrm{F}=$ fibroblast $)$. Morphometric data revealed a similar cell surface area $(A)$ as compared to that of the fibroblasts seeded on TCPS (Figure 6E, $P>0.05$ ). However, the circularity index $(C)$ was less for the fibroblasts cultured on the PGSA as compared to TCPS (Figure 6F, $P<0.05$ ). There was more variety in the morphometric data of the fibroblasts cultured on the PGSA, leading to larger standard deviations than for the fibroblasts cultured on TCPS. The difference of cell attachment on PGSA and TCPS might be related to several factors including surface topography and protein adsorption on the material's surface. The attached cells on the PGSA however proliferated in a linear fashion, forming a confluent cell monolayer at day 12 (Figure 6D). This shows that the in vitro biocompatibility of photocured PGSA allows for cellular attachment and subsequent proliferation into a confluent cell monolayer of primary human fibroblasts.

\section{Conclusions}

We synthesized novel biocompatible, elastomeric biomaterials that are rapidly polymerized at room temperature using UV
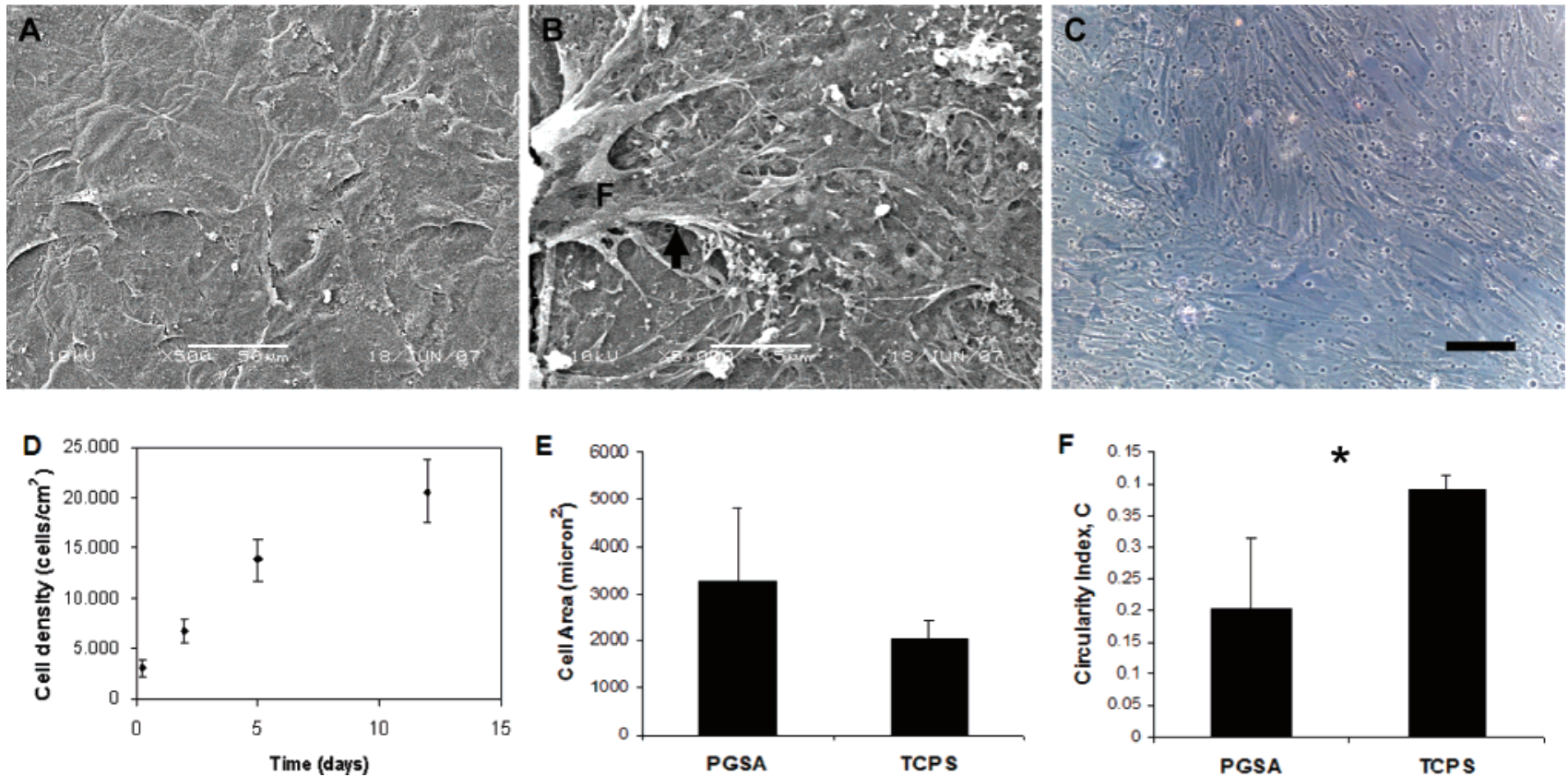

Figure 6. (A) Representative SEM image of the confluent cell monolayer after 12 days of culture on photocured PGSA (DA $=0.34$ ). (B) Representative SEM image of a single fibroblast spreading (arrow) after $24 \mathrm{~h}$ of culture on PGSA (DA $=0.34$ ). $\mathrm{F}=$ fibroblast. (C) Representative phase-contrast image of the confluent cell monolayer after 12 days of culture on photocured PGSA (DA $=0.34$ ) (the black bar represents 100 $\mu \mathrm{m})$. (D) Cell attachment and subsequent proliferation of primary human fibroblasts in vitro on photocured PGSA (DA $=0.34)$. (F) Cell area, $A$, in micrometers squared after $24 \mathrm{~h}$ of culture on PGSA or TCPS. Error bars represent the standard deviation of the mean. $P>0.05$. (F) The circularity of nonconfluent cells was calculated with the equation $C=4(\pi) A / P^{2}$, where $P$ is the perimeter of the cell in micrometers and $A$ is the area of the cell in micrometers squared. Error bars represent the standard deviation of the mean. The asterisk indicates $P<0.05$. 
photopolymerization. These elastomers contain functional hydroxyl groups and exhibit tunable mechanical properties. The liquid acrylated polymer precursor can be combined with other acrylated molecules to further control the physical properties. The development of these novel elastomers could therefore lead to new materials for a variety of potential biomedical applications.

Acknowledgment. C.L.E.N. acknowledges the financial support of Dr. Saal van Zwanenberg Stichting, Vreede Stichting, Shell, and KIVI. J.P.B. acknowledges the J.F.S. Esser Stichting and Stichting Michaël-Van Vloten Fonds for financial support. L.F. acknowledges the financial support of Fundação para a Ciência e a Tecnologia (Grant SFRH/BPD/14502/2003). A.Z. acknowledges financial support from the Swiss National Science Foundation. This work was funded by NIH Grant R01 DE13023, NIH Grant HL060435, and NSF Grant NIRT 0609182.

\section{References and Notes}

(1) Langer, R.; Vacanti, J. P. Tissue engineering. Science 1993, 260 (5110), 920-926.

(2) Wang, Y.; Ameer, G. A.; Sheppard, B. J.; Langer, R. A tough biodegradable elastomer. Nat. Biotechnol. 2002, 20 (6), 602-606.

(3) Pego, A. P.; Poot, A. A.; Grijpma, D. W.; Feijen, J. Biodegradable elastomeric scaffolds for soft tissue engineering. J. Controlled Release 2003, 87, 69-79.

(4) Yang, J.; Webb, A. R.; Ameer, G. A. Novel Citric Acid-Based Biodegradable Elastomers for Tissue Engineering. Adv. Mater. 2004, 16 (6), 511-516.

(5) Gu, F.; Younes, H. M.; El-Kadi, A. O.; Neufeld, R. J.; Amsden, B. G. Sustained interferon-gamma delivery from a photocrosslinked biodegradable elastomer. J. Controlled Release 2005, 102 (3), 607617.

(6) Nasseri, B. A.; Pomerantseva, I.; Kaazempur-Mofrad, M. R.; Sutherland, F. W.; Perry, T.; Ochoa, E.; Thompson, C. A.; Mayer, J. E., Jr.; Oesterle, S. N.; Vacanti, J. P. Dynamic rotational seeding and cell culture system for vascular tube formation. Tissue Eng. 2003, 9 (2), 291-299.

(7) Nugent, H. M.; Edelman, E. R. Tissue engineering therapy for cardiovascular disease. Circ. Res. 2003, 92 (10), 1068-1078.

(8) Belkas, J. S.; Shoichet, M. S.; Midha, R. Peripheral nerve regeneration through guidance tubes. Neurol. Res. 2004, 26 (2), 151-160.

(9) Lundborg, G. Alternatives to autologous nerve grafts. Handchir. Mikrochir. Plast. Chir. 2004, 36 (1), 1-7.

(10) Sundback, C. A.; Shyu, J. Y.; Wang, Y.; Faquin, W. C.; Langer, R. S.; Vacanti, J. P.; Hadlock, T. A. Biocompatibility analysis of poly(glycerol sebacate) as a nerve guide material. Biomaterials 2005, 26, $5454-5464$.

(11) Amsden, B. G.; Tse, M. Y.; Turner, N. D.; Knight, D. K.; Pang, S. C. In vivo degradation behavior of photo-cross-linked star-poly(epsilon-caprolactone-co-D,L-lactide) elastomers. Biomacromolecules 2006, 7 (1), 365-372.

(12) Pego, A. P.; Poot, A. A.; Grijpma, D. W.; Feijen, J. Copolymers of trimethylene carbonate and epsilon-caprolactone for porous nerve guides: synthesis and properties. J. Biomater. Sci., Polym. Ed. 2001, $12(1), 35-53$
(13) Pego, A. P.; Siebum, B.; Van Luyn, M. J.; Gallego, Y.; Van Seijen, X. J.; Poot, A. A.; Grijpma, D. W.; Feijen, J.; Van Luyn, M.; Gallego, Y.; Van Seijen, X. J.; Poot, A. A.; Grijpma, D. W.; Feijen, J. Preparation of degradable porous structures based on 1,3-trimethylene carbonate and D,L-lactide (co)polymers for heart tissue engineering. Tissue Eng. 2003, 9 (5), 981-994.

(14) Wang, Y.; Kim, Y. M.; Langer, R. In vivo degradation characteristics of poly(glycerol sebacate). J. Biomed. Mater. Res., A 2003, 66 (1), 192-197.

(15) Young, K. C.; Main, C.; Gillespie, F. C.; Stephen, K. W. Ultraviolet absorption by two ultra-violet activated sealants. J. Oral Rehabil. 1978, 5 (3), 207-13.

(16) Venhoven, B. A.; de Gee, A. J.; Davidson, C. L. Light initiation of dental resins: dynamics of the polymerization. Biomaterials 1996, 17 (24), 2313-2318.

(17) Leonard, D. L.; Charlton, D. G.; Roberts, H. W.; Cohen, M. W. Polymerization efficiency of LED curing lights. J Esthet. Restor. Dent. 2002, 14 (5), 286-295.

(18) West, J. L.; Hubbell, J. A. Comparison of covalently and physically cross-linked polyethylene glycol-based hydrogels for the prevention of postoperative adhesions in a rat model. Biomaterials 1995, 16 (15), 1153-1156.

(19) Elisseeff, J.; McIntosh, W.; Anseth, K.; Riley, S.; Ragan, P.; Langer, R. Photoencapsulation of chondrocytes in poly(ethylene oxide)-based semi-interpenetrating networks. J. Biomed. Mater. Res., A 2000, 51 (2), 164-171.

(20) Cruise, G. M.; Scharp, D. S.; Hubbell, J. A. Characterization of permeability and network structure of interfacially photopolymerized poly(ethylene glycol) diacrylate hydrogels. Biomaterials 1998, 19 (14), 1287-1294.

(21) Anseth, K. S.; Burdick, J. A. New directions in photopolymerizable biomaterials. MRS Bull. 2002, 27(2), 130-136.

(22) Ferreira, L.; Gil, M. H.; Cabrita, A. M.; Dordick, J. S. Biocatalytic synthesis of highly ordered degradable dextran-based hydrogels. Biomaterials 2005, 26 (23), 4707-4716.

(23) Tsujimoto, K.; Uyama, H.; Kobayashi, S. Enzymatic Synthesis and Curing of Biodegradable Crosslinkable Polyesters. Macromol. Biosci. 2002, 2 (7), 329-335.

(24) Yang, Y. S.; Qi, G. R.; Qian, J. W.; Yang, S. L. Acryloyl Chloride Polymer. J. Appl. Polym. Sci. 1998, 68, 665-670.

(25) Rydevik, B. L.; Kwan, M. K.; Myers, R. R.; Brown, R. A.; Triggs, K. J.; Woo, S. L.; Garfin, S. R. An in vitro mechanical and histological study of acute stretching on rabbit tibial nerve. J. Orthop. Res. 1990, 8 (5), 694-701.

(26) Yang, J.; Webb, A. R.; Pickerill, S. J.; Hageman, G.; Ameer, G. A. Synthesis and evaluation of poly(diol citrate) biodegradable elastomers. Biomaterials 2006, 27 (9), 1889-1898.

(27) Woo, G. L.; Mittelman, M. W.; Santerre, J. P. Synthesis and characterization of a novel biodegradable antimicrobial polymer. Biomaterials 2000, 21 (12), 1235-1246.

(28) Tang, Y. W.; Labow, R. S.; Santerre, J. P. Enzyme induced biodegradation of polycarbonate-polyurethanes: dose dependence effect of cholesterol esterase. Biomaterials 2003, 24 (12), 20032011

(29) Santerre, J. P.; Woodhouse, K.; Laroched, G.; Labow, R. S. Understanding the biodegradation of polyurethanes: from classical implants to tissue engineering materials. Biomaterials 2005, 26 (35), $7457-7470$

BM070423U 